\title{
Severe Hypertriglyceridemia Possibly Masked Acute Pancreatitis and Led to a Difficult Diagnosis in an Obese Patient with Ketoacidosis-onset Type 2 Diabetes
}

\author{
Midori Fujishiro ${ }^{1}$, Akiko Horita ${ }^{1}$, Hiroshi Nakagawara ${ }^{2}$, Takayuki Mawatari ${ }^{3}$, \\ Yoshifusa Kishigami ${ }^{4}$, Yoshiteru Tominaga ${ }^{3}$, Mitsuhiko Moriyama ${ }^{2}$ and Hisamitsu Ishihara ${ }^{1}$
}

\begin{abstract}
:
A young obese man with ketoacidosis-onset type 2 diabetes mellitus, associated with severe hypertriglyceridemia, was admitted to a local hospital complaining of abdominal pain. Although the abdominal pain worsened, his serum amylase level remained normal with persistent severe hypertriglyceridemia until the second day of hospitalization. The next day, computed tomography showed severe acute pancreatitis (AP) with serum amylase elevation, while the patient's triglyceride level decreased to $558 \mathrm{mg} / \mathrm{dL}$. He was transferred to our hospital and recovered after intensive care. AP accompanied by diabetic ketoacidosis is not rare but an early diagnosis can be difficult to make due to normal amylase levels in the presence of severe hypertriglyceridemia.
\end{abstract}

Key words: Type 2 diabetes mellitus, diabetic ketoacidosis, hypertriglyceridemia, pancreatitis, amylase activity, abdominal pain

(Intern Med 56: 2611-2616, 2017)

(DOI: 10.2169/internalmedicine.8474-16)

\section{Introduction}

Diabetic ketoacidosis (DKA) is characterized by the triad of hyperglycemia, metabolic acidosis and ketonemia, resulting from absolute or relative insulin deficiency. It represents the most serious hyperglycemic emergency in patients with diabetes. In general, DKA is a hallmark of type 1 diabetes mellitus $(1,2)$, but it can also occur in type 2 diabetes mellitus (T2DM), as seen in patients of African or Hispanic descent $(3,4)$. In recent years, an increasing number of T2DM patients with ketoacidosis has also been reported in Japan $(5,6)$. Nagasaka et al. reported that these patients were characterized by their young age (18-23 years), male sex, obesity [body mass index (BMI), 25.4-44.5 kg/m²], a positive family history of T2DM and anti-glutamic acid decarboxylase (GAD) antibody negativity, together with the overconsumption of soft drinks (6). The prevalence of DKA in
Japan may reflect the large numbers of soft drink vending machines in the country, as well as DKA-prone young obese people slipping through the Japanese health check-up system, which targets individuals of 40-74 years of age with "obesity diseases" or "metabolic syndrome" and encourages their participation in lifestyle modification programs (7). In many cases, these young people are unaware that they are prone to diabetes or that may have already developed diabetes, and as such do not refrain from consuming sugarcontaining soft drinks to quench their thirst, a symptom of hyperglycemia, which can lead to ketoacidosis.

DKA in obese T2DM patients is often associated with hypertriglyceridemia (HTG), which is caused by relative insulin deficiency and/or by obesity itself $(8,9)$. These conditions often lead to acute pancreatitis (AP), which is a lifethreatening disease (10-16). Since DKA is frequently associated with abdominal pain, particularly if the degree of acidosis is severe (17), physicians should be aware that their

${ }^{1}$ Division of Diabetes and Metabolic Diseases, Nihon University School of Medicine, Japan, ${ }^{2}$ Department of Gastroenterology and Hepatology, Nihon University Hospital, Japan, ${ }^{3}$ Division of Emergency and Critical Care Medicine, Department of Acute Medicine, Nihon University Hospital, Japan and ${ }^{4}$ Department of Internal Medicine, Sonoda Daiichi Hospital, Japan

Received: October 30, 2016; Accepted: February 20, 2017; Advance Publication by J-STAGE: September 6, 2017

Correspondence to Dr. Midori Fujishiro, fujishiro.midori@nihon-u.ac.jp 


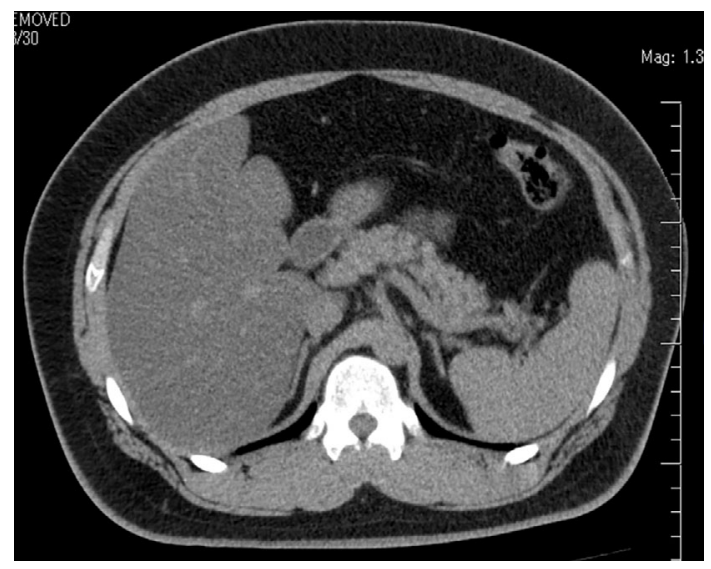

Figure 1. The CT scan obtained on admission to the referring hospital showed no noteworthy abnormalities.

abdominal pain may be caused by the DKA itself or by accompanying pancreatitis. When hyperlipidemia is also present, both serum and urine amylase examinations may yield false-normal levels (18). Physicians evaluating such patients might be misled by these seemingly normal serum amylase values and thereby exclude pancreatitis from the differential diagnoses.

We managed a young obese Japanese man who presented with ketoacidosis-onset T2DM with abdominal pain and HTG. Initially, pancreatitis could not be detected because of essentially normal computed tomography (CT) findings and a normal serum amylase level. Although DKA treatment was initiated, his abdominal pain persisted and worsened while his serum amylase remained normal on the second day of hospitalization; his HTG remained severe at this time point. On the third day of hospitalization, with the improvement of HTG, the elevated amylase level became apparent and CT revealed changes typical of severe pancreatitis. His AP was successfully treated and he was discharged with prescriptions for an oral triglyceride-lowering agent and an oral insulin-sensitizing agent.

\section{Case Report}

A 25-year-old man, who was previously healthy but was recognized as having fatty liver associated with mild hepatic dysfunction, visited a local doctor complaining of persistent nausea and abdominal pain. His blood test results revealed DKA with the following findings: plasma glucose, $429 \mathrm{mg} /$ dL; HbA1c, 10.7\%; total ketone bodies, 7,850 $\mu \mathrm{mol} / \mathrm{L}$ and arterial $\mathrm{pH}, 7.22$. He was transferred to a local emergency hospital by ambulance. On admission, CT showed no serious abnormalities (Fig. 1). His serum amylase level was normal (35 U/L) but his serum HTG was severe $(1,852 \mathrm{mg}$ / $\mathrm{dL})$, resulting in lactescent serum. Although DKA treatment was initiated, his abdominal pain persisted and worsened while his serum amylase level remained normal (39 U/L). On the second day of hospitalization, the patient's HTG remained severe $(1,252 \mathrm{mg} / \mathrm{dL})$. On the third day of hospitali-

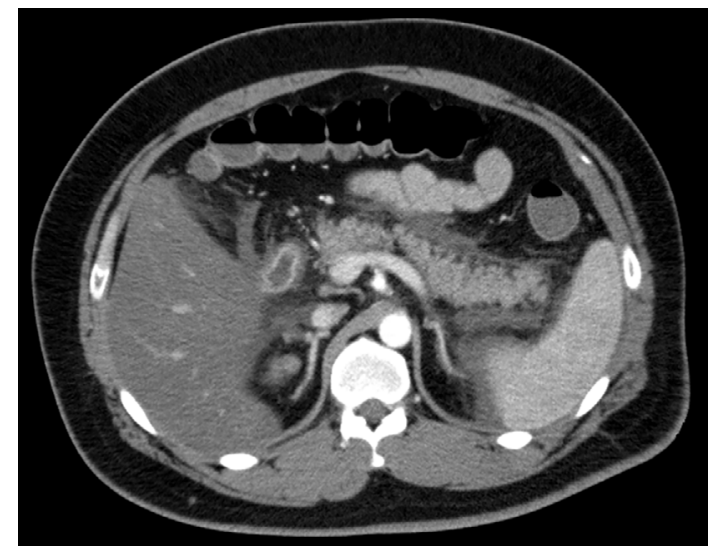

Figure 2. The second CT scan obtained at the referring hospital showed acute inflammatory changes, with swelling of not only the pancreas but also around the spleen, and ascites.

zation, his abdominal pain markedly worsened. On that day, his serum triglyceride level had decreased to $558 \mathrm{mg} / \mathrm{dL}$ with no signs of chylomicronemia; however, his serum amylase level had risen to $666 \mathrm{U} / \mathrm{L}$. Abdominal CT was repeated and showed AP (Fig. 2). Fig. 3 shows the extra-pancreatic progression of inflammation extending beyond the lower pole of the kidney, which counts as 2 points resulting in CT Grade of 2. The severity was graded as "severe" according to the latest Japanese severity scoring system, which was proposed by the Research Committee of Intractable Pancreatic Diseases (RCIPD) in 2008. The prognostic factors and the contrast-enhanced CT grade are prepared for the system proposed by the RCIPD such that severity can be assessed according to both criteria (19). The patient was transferred to our hospital in an ambulance equipped with an intensive care unit. He was conscious with a Glasgow Coma Scale score of 15 points (E4V5M6). His vital signs were relatively stable with a body temperature of $37.6^{\circ} \mathrm{C}$, a blood pressure of $139 / 94 \mathrm{mmHg}$, a regular pulse of 113 beats/min and an oxygen saturation of $98 \%$ on $3 \mathrm{~L} / \mathrm{min}$ of oxygen by nasal cannula. A physical examination revealed no evidence of xanthoma or xanthelasma. No palpable lymph node enlargement was detected in a head and neck examination. On abdominal examination, there was tenderness in the left lateral region with muscular defense and Blumberg's sign.

An arterial blood gas analysis revealed the following: $\mathrm{pH}$, 7.23; carbon dioxide partial pressure $\left(\mathrm{PaCO}_{2}\right), 26.3 \mathrm{mmHg}$; partial pressure oxygen $\left(\mathrm{PaO}_{2}\right), 172.1 \mathrm{mmHg} ; \mathrm{HCO}_{3}-10.6$ $\mathrm{mEq} / \mathrm{L}$; BE, $-15.0 \mathrm{mEq} / \mathrm{L}$; and saturation of arterial oxygen $\left(\mathrm{SaO}_{2}\right), 99.4 \%$ on $3 \mathrm{~L} / \mathrm{min} \mathrm{O}_{2}$ by nasal cannula. Ketones were present in the urine $(3+$, as measured by sodium nitroprusside reaction). The patient's HbA1c level was $11.3 \%$ but his glucose and triglyceride levels were reduced to $271 \mathrm{mg} /$ $\mathrm{dL}$ and $388 \mathrm{mg} / \mathrm{dL}$, respectively, with DKA treatment (Table). The total bilirubin value of $0.75 \mathrm{mg} / \mathrm{dL}$ and the aspartate aminotransferase (AST) value of $31 \mathrm{U} / \mathrm{L}$ were normal; however, his alanine aminotransferase (ALT) level was elevated to $139 \mathrm{U} / \mathrm{L}$ and his gamma $\gamma$-glutamyl transpeptidase (GTP) level was elevated to $119 \mathrm{U} / \mathrm{L}$, suggesting fatty liver. 

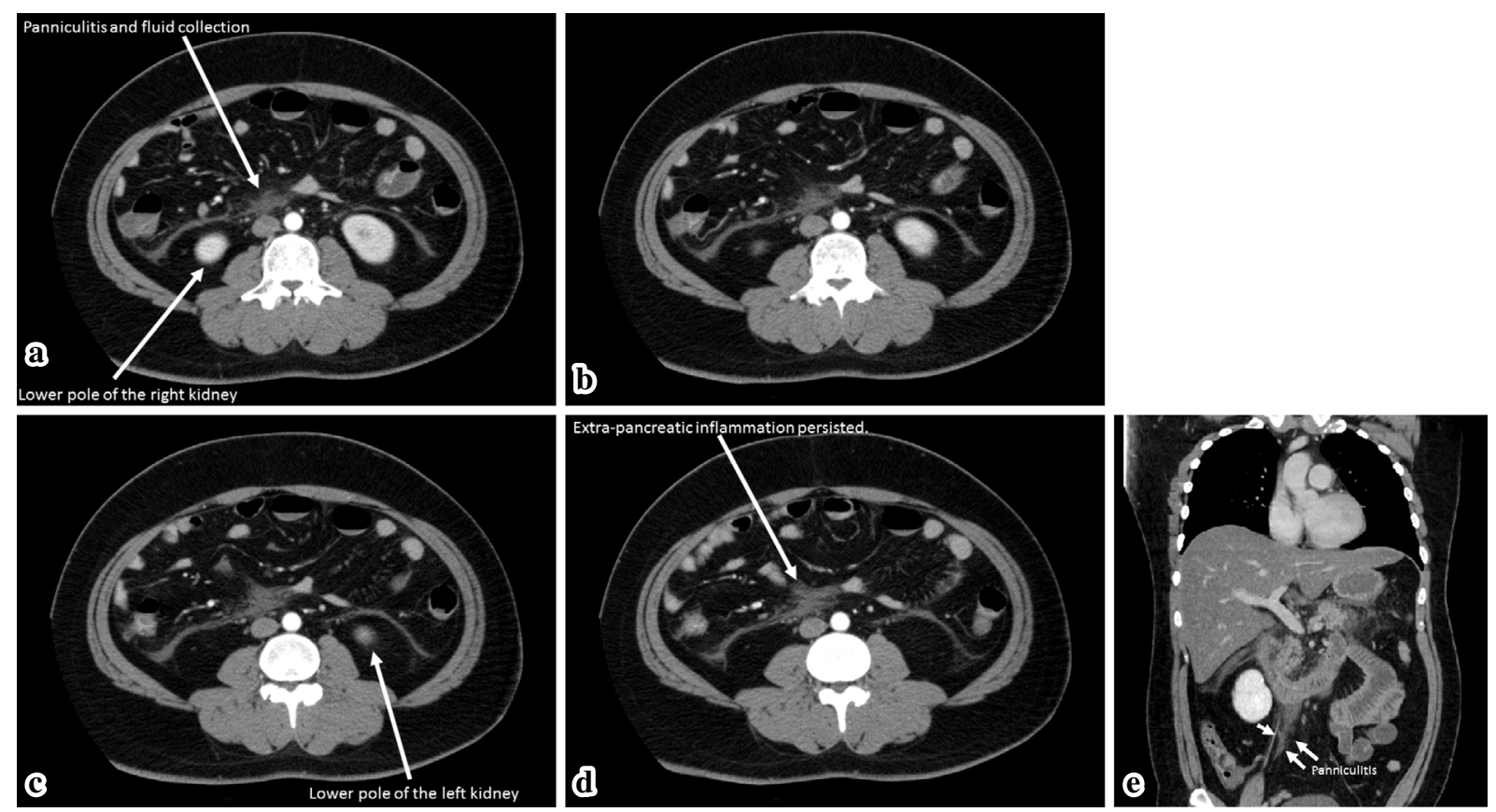

Figure 3. Slices from the second CT scan showing the area around the lower poles of the bilateral kidneys, which provided evidence of the extra-pancreatic progression of inflammation beyond the lower pole of the kidney. (a) A slice from the second CT scan showing the lower pole of the right kidney with panniculitis and fluid collection. (b and c) Slices from the second CT scan obtained in the area around the lower pole of the left kidney with panniculitis and fluid collection. (d) A slice from the second CT shows the area under the lower pole of the kidney and confirms the presence of extrapancreatic inflammation. (e) A coronal reconstruction of the second CT obtained in the area around the lower pole of the right kidney with panniculitis.

The results of the other laboratory data were as follows; white cell count, 9,500/ $\mathrm{LL}$; hemoglobin, $15.1 \mathrm{~g} / \mathrm{dL}$; Creactive protein $(\mathrm{CRP}), 28.04 \mathrm{mg} / \mathrm{dL}$; amylase, $582 \mathrm{U} / \mathrm{L}$; lipase, 1,415 U/L; elastase-1, 6,490 ng/dL; trypsin, 9,070 ng/ $\mathrm{mL}$; pancreatic phospholipase, $\mathrm{A} 1,5,190 \mathrm{ng} / \mathrm{mL}$; albumin, $3.5 \mathrm{~g} / \mathrm{dL}$; calcium, $8.5 \mathrm{mg} / \mathrm{dL}$; sodium, $131 \mathrm{mEq} / \mathrm{L}$; potassium, $4.1 \mathrm{mEq} / \mathrm{L}$; and chloride, $104 \mathrm{mEq} / \mathrm{L}$.

The patient's uncle was diabetic but he did not know whether there was a family history of hyperlipidemia. He had never consumed alcohol, nor had he previously experienced either pancreatitis or gallstone disease. He had been engaged in the maintenance and safety checking of elevators until 5 months prior to his admission, when he quit his job. He had eaten as much as he wanted, including steaks and hamburgers for dinner, followed by Chinese noodles. During the 10 days prior to the present event, he had consumed at least 2 liters of sugar-containing soft drinks per day. Although he weighed $116 \mathrm{~kg}$ (BMI $\left.39.2 \mathrm{~kg} / \mathrm{m}^{2}\right)$ at 1 month before admission, he had lost $16 \mathrm{~kg}$ before visiting the hospital. Thus, on admission, his height was $172 \mathrm{~cm}$ and he weighed $100 \mathrm{~kg}$ (BMI was $33.8 \mathrm{~kg} / \mathrm{m}^{2}$ ).

After being transferred to our hospital, his body temperature increased to $>38^{\circ} \mathrm{C}$. By then, he met 3 of 9 prognostic factors: $\mathrm{BE} \leq 3 \mathrm{mEq} / \mathrm{L}$ (factor 1 ), $\mathrm{CRP} \geq 15 \mathrm{mg} / \mathrm{dL}$ (factor 7), and number of positive systemic inflammatory response syndrome (SIRS) criteria $\geq 3$ (factor 8 ), such as temperature $>$ $38^{\circ} \mathrm{C}$, heart rate $>90$ beats $/ \mathrm{min}$, and both the respiratory rate
$>20$ breaths/min and $\mathrm{PaCO}_{2}<32 \mathrm{mmHg}$. His AP was evaluated as "severe" according to the criteria for prognostic factors proposed by the RCIPD as well as the CT grade (19). It is difficult to determine the main factor accounting for the negative BE levels in AP patients with accompanying DKA (as in our case) because DKA itself induces negative BE values. In our case, the patient's negative BE levels persisted in spite of the treatment of DKA, along with the gradual lowering of blood glucose level and serum TG levels, which suggests that the negative BE level on the third day of hospitalization was mainly due to severe AP. At any rate, the patient had already been diagnosed with severe AP according to the CT grade, independently of the prognostic score.

The patient's DKA was treated with intravenous hydration using normal saline, potassium supplementation, and intravenous regular insulin. The AP was managed with intravenous hydration, a protease inhibitor, antibiotics and a proton pump inhibitor. When the patient's acidosis was corrected, the ketosis had fully resolved, and the patient had recovered enough to eat, intravenous insulin infusion was shifted to multiple subcutaneous injections, together with bezafibrate (400 mg, orally, twice a day). After the patient's inflammation was controlled (confirmed by the reduced serum CRP level), his 24-hour urine C-peptide immunoreactivity value was $149 \mathrm{U} \mu \mathrm{L} /$ day and was negative for anti-GAD antibody. He was then started on metformin (an oral hypoglycemic agent) followed by the gradual tapering and eventual discon- 
Table. Laboratory Values (Numbers in Bold Type are Outside of the Reference Range).

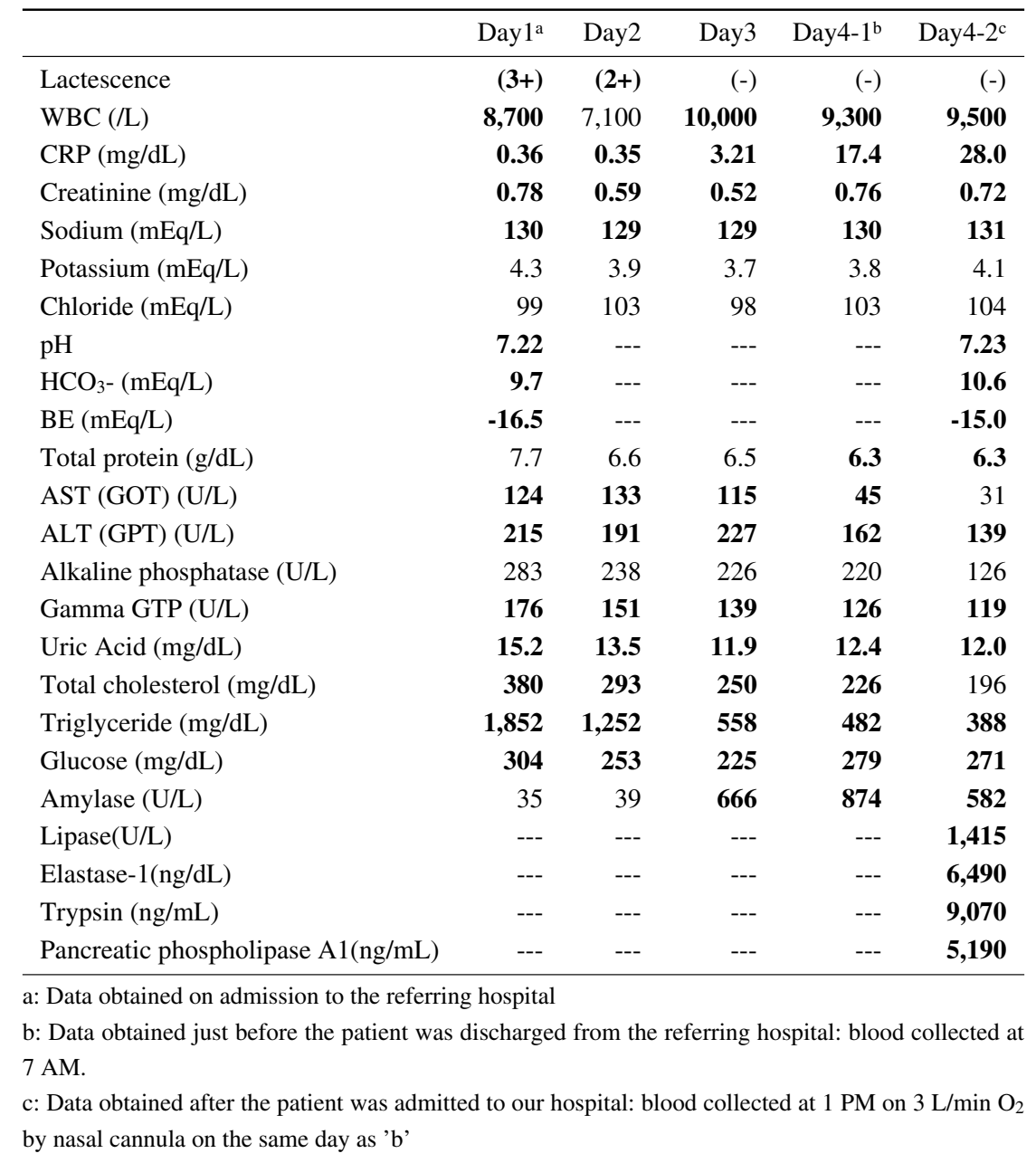

tinuation of insulin injections. The patient was given instructions to implement a calorie and fat restricted diet by a nutritionist and was discharged on the 34th day of hospitalization with fasting glucose and triglyceride levels of $84 \mathrm{mg} / \mathrm{dL}$ and $306 \mathrm{mg} / \mathrm{dL}$, respectively. He is currently being followed by a local physician with prescriptions for metformin and bezafibrate.

\section{Discussion}

Abdominal pain and tenderness are common in patients with severe DKA. According to Campbell et al., unexplained abdominal pain associated with severe ketoacidosis is indicated by a plasma bicarbonate level of $\leq 10 \mathrm{mEq} /$ L (17). The arterial blood gas analysis of our patient, which was initially obtained at the referring hospital showed a plasma bicarbonate level of $9.7 \mathrm{mEq} / \mathrm{L}$ and his abdominal pain could only be explained as being due to severe ketoacidosis.

AP reportedly occurs in at least $10 \%$, but possibly as many as $15 \%$, of DKA patients (10). The pathogenesis of AP in DKA varies but-importantly-transient and profound hyperlipidemia is an identifiable causative factor. However, as a number of reports have highlighted $(18,20,21)$, clini- cal problems are often encountered because severe HTG can mask other laboratory measurements. The massive accumulation of triglycerides interferes with the determination of biochemical values and results in seemingly normal or even low levels of electrolytes. In fact, even glucose concentrations can be falsely low in the presence of HTG. In some cases, the diagnosis and treatment of DKA may be delayed due to pseudonormoglycemia in the presence of massively elevated serum triglyceride levels (20). With regard to amylase, on occasion, both the serum and urinary amylase levels in hypertriglyceridemic patients with pancreatitis are normal, and show no marked elevation (21), which may hamper making an accurate diagnosis of AP. Despite the seemingly low amylase activities in the serum and urine, the amylase/ creatinine clearance ratio rises in hypertriglyceridemic patients with pancreatitis and its measurement thus constitutes a potentially effective diagnostic aid (18). It has been suggested that there is a circulating inhibitor of amylase in the serum of many patients with HTG-associated pancreatitis (21). The origin and nature of this amylase inhibitor remain unknown but urinary filtration is likely responsible. The clinical importance of the inhibition of amylase activity lies in its capacity to steer physicians away from the diagnosis of AP. 
The most common etiologies of AP include gallstone disease and alcohol abuse (22), neither of which contributed in the present case. HTG is also a well-known cause of AP. HTG was considered to be a causative factor in $1-10 \%$ of the patients in reports on $\operatorname{AP}(8,23)$. Overweight or obese patients are reportedly prone to AP, which might be associated with the more severe insulin resistance and poorer glycemic control that accompany HTG (9).

AP can be difficult to diagnose without high serum amylase levels. It is important to be aware that abdominal pain in a patient with lactescent serum suggests pancreatitis, even if the serum amylase levels are apparently normal. Our patient presented with abdominal pain accompanied by HTG and normal serum amylase levels, which persisted through the second day of hospitalization, possibly pointing away from a diagnosis of AP. In DKA patients with abdominal pain, it is prudent not to ignore AP as a clinical component of DKA. Rather physicians should obtain further amylase, lipase, and triglyceride measurements. A CT scan of the abdomen should be performed if AP is suspected. Furthermore, as previously reported, the correction of the amylase activity by dilution (2-16-fold) and appropriate recalculation can unmask the "true" state of hyperamylasemia (24).

Unfortunately, we cannot confirm that the onset of our patient's AP occurred before the third day of hospitalization (when the diagnosis was made), because we did not carry out further examinations to diagnose AP prior to that day. It is possible that the initial symptom of abdominal pain on day 1 was not attributable to AP. However, our patient's AP might have started on the second day of hospitalization (the day before the diagnosis of AP was confirmed). Unfortunately, we have no evidence to support this hypothesis. However, it seems reasonable since the patient's abdominal pain worsened markedly on that day, despite treatment for DKA-this raised the suspicion of another concurrent disease rather than a worsening of the DKA state.

As in our present case, even with an acute presentation of severe insulin deficiency and ketoacidosis accompanied by severe pancreatitis, when AP is accurately diagnosed and appropriately managed, the majority of newly diagnosed obese adult T2DM patients can discontinue insulin therapy during follow-up and remain in near-normoglycemic remission without insulin for several months to years (25). As Valdivielso et al. indicated, once the HTG-AP episode has resolved, the prevention of the next episode is essential (26). They emphasized that lifestyle changes and dietary modifications are the keys to the long-term management of HTG. They advocated dietary education from a nutritionist for patients who have suffered from an episode of HTG-associated AP (26), and we followed this advice in the management of our patient.

Non-specific abdominal pain is common in patients with severe DKA. Furthermore, DKA in obese T2DM patients is often associated with HTG and on occasion becomes severe. Severe HTG may lead to seemingly normal or even low levels of several biochemical values, including amylase-making it difficult to diagnose AP at its onset. Clinicians should be mindful of the possibility of associated AP and pay particular attention to complaints of abdominal pain-despite nonspecific abdominal pain being relatively common-even if serum amylase levels are normal. Because AP represents a life-threatening disease, an accurate diagnosis and the prompt initiation of treatment are very important. If a DKA patient has lactescent serum, correcting the serum amylase activity measurement by dilution and a CT scan of the abdomen will be helpful for making a correct diagnosis of AP.

The authors state that they have no Conflict of Interest (COI).

\section{References}

1. Umpierrez GE, Khajavi M, Kitabchi AE. Review: diabetic ketoacidosis and hyperglycemic, hyperosmolar nonketotic syndrome. Am J Med Sci 311: 225-233, 1996.

2. Delaney MF, Zisman A, Kettyle WM. Diabetic ketoacidosis and hyperglycemic hyperosmolar nonketotic syndrome. Endocrinol Metab Clin North Am 29: 683-705,V, 2000.

3. Umpierrez GE, Smiley D, Kitabchi AE. Narrative review: ketosisprone type 2 diabetes mellitus. Ann Int Med 144: 350-357, 2006.

4. Umpierrez GE, Casals MM, Gebhart SP, et al. Diabetic ketoacidosis in obese African-Americans. Diabetes 44: 790-795, 1995.

5. Yamada K, Nonaka K. Diabetic ketoacidosis in young obese Japanese men. Diabetes Care 19: 671, 1996.

6. Nagasaka S, Ishikawa $\mathrm{S}$, Itabashi $\mathrm{N}$, et al. Ketoacidosis-onset type 2 diabetes in Japanese. Association with the widespread distribution of soft drinks and vending machines. Diabetes Care 21: 13761378, 1998.

7. Mizushima S, Tsushita K. New strategy on prevention and control of noncommunicable lifestyle-related diseases focusing on metabolic syndrome in Japan. In: Asian Perspectives and Evidence on Health Promotion and Education. Muto T, Nam EW, Nakahara T, Eds. Springer Japan, Tokyo, 2011: 31-39.

8. Fortson MR, Freedman SN, Webster PD 3rd. Clinical assessment of hyperlipidemic pancreatitis. Am J Gastroenterol 90: 2134-2139, 1995.

9. Fernandez R, Misra R, Nalini R, et al. Characteristics of patients with ketosis-prone diabetes (KPD) presenting with acute pancreatitis: implications for the natural history and etiology of a KPD subgroup. Endocr Pract 19: 243-251, 2013.

10. Nair S, Yadav D, Pitchumoni CS. Association of diabetic ketoacidosis and acute pancreatitis: observations in 100 consecutive episodes of DKA. Am J Gastroenterol 95: 2795-2800, 2000.

11. Yamaguchi K, Kayashima T, Hashiguchi Y. Painless acute pancreatitis associated with diabetic ketoacidosis in a case of juvenile onset obese non-insulin-dependent diabetes mellitus. J Jpn Diab Soc 42: 163-168, 1999 (in Japanese, Abstract in English).

12. Soejima S, Umeno Y, Fujita T, Dohmen K, Miyamoto Y. A case of diabetic ketoacidosis complicated by severe hypertriglyceridemia and acute pancreatitis. J Jpn Diab Soc 43: 561-566, 2000 (in Japanese, Abstract in English).

13. Tanaka M, Miyazaki Y. Diabetic ketoacidosis induced by excessive intake of soft drink complicated by hypercalcemia, hypertriglyceridemia and painless acute pancreatitis in a case of type 2 diabetes mellitus. J Jpn Diab Soc 44: 913-916, 2001 (in Japanese).

14. Yamamoto $K$, Yanagimoto S, Kubota A, et al. A case of diabetic ketoacidosis with severe pancreatitis and marked hypertriglyceridemia successfully treated with plasma exchange. J Jpn Diab Soc 53: 613-618, 2010 (in Japanese).

15. Yamamoto S, Okada Y, Arao T, Nishida K, Tanaka Y. A case of 
severe acute pancreatitis associated with hypertriglyceridemia caused by soft drink ketoacidosis. J Jpn Diab Soc 54: 34-39, 2011 (in Japanese).

16. Hosokawa Y, Fukumoto M, Yoshida Y, et al. A case of hypertriglyceridemic pancreatitis during pregnancy in a patient with type 2 diabetes. J Jpn Diab Soc 57: 113-117, 2014 (in Japanese, Abstract in English).

17. Campbell IW, Duncan LJ, Innes JA, et al. Abdominal pain in diabetic metabolic decompensation. Clinical significance. JAMA 233: 166-168, 1975.

18. Lesser PB, Warshaw AL. Diagnosis of pancreatitis masked by hyperlipemia. Ann Int Med 82: 795-798, 1975.

19. Takeda K, Yokoe M, Takada T, et al. Assessment of severity of acute pancreatitis according to new prognostic factors and CT grading. J Hepatobiliary Pancreat Sci 17: 37-44, 2010.

20. Kaminska ES, Pourmotabbed G. Spurious laboratory values in diabetic ketoacidosis and hyperlipidemia. Am J Emerg Med 11: $77-$ 80, 1993.

21. Warshaw AL, Bellini CA, Lesser PB. Inhibition of serum and urine amylase activity in pancreatitis with hyperlipemia. Ann Surg
182: $72-75,1975$.

22. Yadav D, Lowenfels AB. Trends in the epidemiology of the first attack of acute pancreatitis: a systematic review. Pancreas 33: 323330, 2006.

23. Winslet M, Hall C, London NJ, et al. Relation of diagnostic serum amylase levels to aetiology and severity of acute pancreatitis. Gut 33: 982-986, 1992.

24. Fallat RW, Vester JW, Glueck CJ. Suppression of amylase activity by hypertriglyceridemia. JAMA 225: 1331-1334, 1973.

25. Smiley D, Chandra P, Umpierrez GE. Update on diagnosis, pathogenesis and management of ketosis-prone Type 2 diabetes mellitus. Diabetes Manag (Lond) 1: 589-600, 2011.

26. Valdivielso P, Ramírez-Bueno A, Ewald N. Current knowledge of hypertriglyceridemic pancreatitis. Eur J Intern Med 25: 689-694, 2014.

The Internal Medicine is an Open Access article distributed under the Creative Commons Attribution-NonCommercial-NoDerivatives 4.0 International License. To view the details of this license, please visit (https://creativecommons.org/licenses/ by-nc-nd/4.0/).

(C) 2017 The Japanese Society of Internal Medicine

Intern Med 56: 2611-2616, 2017 